\title{
Sob o Espectro da Neurociência: a Neurossociologia, a Psicologia Social e as Abordagens Biossociais
}

\author{
André Luís Ribeiro Lacerda ${ }^{1}$ \\ ${ }^{1}$ Universidade Federal do Mato Grosso, Cuiabá, MT, Brasil.
}

\begin{abstract}
Resumo: O desenvolvimento da neurociência tem recebido atenção das diferentes especialidades que formam o campo disciplinar da sociologia? Para responder a essa pergunta, formulou-se a hipótese de que duas especialidades sociológicas poderiam estar fomentando recombinações com especialidades da neurociência, a neurossociologia, nascente disciplina, produto de recombinações de especialidades da sociologia com especialidades da neurociência, e a sociologia cognitiva. Para verificar a hipótese, optou-se por realizar uma revisão da literatura, porintermédio de revisão sistemática, um processo padronizado de busca em três bases de dados, o que poderia indicar se a produção da literatura nas duas especialidades tem conversado com a neurociência. Os vinte cinco textos encontrados mostraram que as duas principais especialidades sociológicas que dialogam com a neurociência são a neurossociologia e a psicologia social sociológica, seguidas da teoria social e da sociologia cognitiva. A neurossociologia tem laços sociais fortes com a sociologia evolucionista, campo mais periférico, enquanto a psicologia social é uma especialidade mais tradicional e, talvez por isso, a neurossociologia tem interagido com a neurociência a partir de recombinações com especialidades da biologia, enquanto a psicologia social parece reticente em percorrer esse caminho. Essas diferenças de percurso ajudam a entender as temáticas e os conceitos explorados nas duas especialidades. Novas investigações poderão sustentar se essas tendências serão perpetuadas.
\end{abstract}

Palavras-chave: Neurociência, Revisão Sistemática, Neurossociologia, Psicologia Social.

\section{Under the Neuroscience Spectrum: Neurosociology, Social Psychology and Biosocial Approaches}

\begin{abstract}
Has the development of neuroscience received attention from the different specialties assembling the field of sociology? To answer this question, this study formulated the hypothesis that two sociological specialties could be promoting a recombination with neuroscience specialties, namely: (1) neurosociology, an emerging discipline arising from the recombination of areas of specialization within sociology and neuroscience; and (2) cognitive sociology. Such hypothesis was verified by means of a systematic literature review conducted in three databases, analyzing whether the academic production in the two specialties has referred to neuroscience. The twenty-five texts found show that the two main sociological specialties dialoguing with neuroscience are neurosociology and sociological social psychology, followed by social theory and cognitive sociology. Whereas neurosociology is strongly associated with evolutionary sociology - a more peripheral field, - social psychology is a more traditional specialty, which may explain why neurosociology has interacted with neuroscience from the recombination with biology specialties while social psychology seems reticent to follow that path. Such differences help understand the issues and concepts explored in these two specialties. Further investigations may clarify whether these trends will continue.
\end{abstract}

Keywords: Neuroscience, Systematic Review, Neurosociology, Social Psychology. 


\title{
Bajo el Espectro de la Neurociencia: Neurosociología, Psicología Social y Enfoques Biosociales
}

\begin{abstract}
Resumen: ${ }_{i}$ El desarrollo de la neurociencia ha recibido atención de las diferentes especialidades que forman el campo disciplinario de la sociología? Para responder a esta pregunta, se formuló la hipótesis de que dos especialidades sociológicas podrían promover la recombinación con especialidades de la neurociencia: la neurosociología, una nueva disciplina producto de la recombinación de especialidades de la sociología con especialidades de la neurociencia, y la sociología cognitiva. Para verificar esta hipótesis, se decidió llevar a cabo una revisión de la literatura, por medio de un proceso de búsqueda estandarizado, la revisión sistemática en tres bases de datos, lo que podría indicar si la producción de la literatura en las dos especialidades ha dialogado con la neurociencia. Los veinticinco textos encontrados mostraron que las dos especialidades sociológicas principales que dialogan con la neurociencia son la neurosociología y la psicología social sociológica, seguidas más tarde por la teoría social y la sociología cognitiva. La neurosociología tiene fuertes lazos sociales con la sociología evolutiva, un campo más periférico, mientras que la psicología social es una especialidad más tradicional y, quizás debido a esto, la neurosociología ha interactuado con la neurociencia a partir de la recombinación con especialidades de biología mientras la psicología social parece reticente a ir por ese camino. Estas diferencias de ruta ayudan a comprender los temas y conceptos explorados en las dos especialidades. Más investigaciones pueden confirmar si estas tendencias continuarán.
\end{abstract}

Palabras clave: Neurociencia, Revisión Sistemática, Neurosociología, Psicología Social.

\section{Introdução}

Em um célebre estudo sobre a sociologia norte-americana, Turner e Turner (1990) mostraram a diversidade do campo profissional, sua falta de paradigmas unificadores e sua ampla e eclética agenda de pesquisa. Entre outras coisas, descreveram a estrutura social das especialidades sociológicas. Hoje, a Associação Americana de Sociologia tem 52 seções, que representam grupos de sociólogos que compartilham interesses comuns em um tópico específico e abordam tópicos que variam do envelhecimento à globalização. Pode-se chamar as seções de especialidades, na medida em que essas seções são nomeadas pelas próprias especialidades.

O desenvolvimento de especialidades e subespecialidades em qualquer disciplina científica é variado e desigual. Sther (1975), por exemplo, mostrou que o crescimento médio do número de sociólogos representava a soma de tendências muito diferentes nas variadas especialidades sociológicas. Conforme bem pontuado por ele, o desenvolvimento do domínio cognitivo e estrutural de uma disciplina e suas especialidades não ocorre "no vácuo", na medida em que, semelhantemente ao que ocorre em outras estruturas sociais, existem mudanças na composição dos membros de uma especialidade. Algumas especialidades têm mais prestígio e mais recursos, ao contrário de outras, assim como algumas enfrentam mais resistência, seja teórica, seja ideológica.

Segundo Sther (1975), o desenvolvimento desigual de especialidades está sistematicamente relacionado não apenas aos aspectos cognitivos e características estruturais da própria especialidade, de outras especialidades, da própria disciplina e de outras disciplinas, mas também às condições sociais em geral. A sócio lógica do argumento de Sther (1975) foi baseada na observação empírica de taxas variadas de crescimento dos membros de diferentes áreas de especialização da sociologia. Sther e Larson (1972) mostraram que as áreas de especialização dos sociólogos haviam mudado significativamente entre 1950 e 1970. Em 1972, especialidades como organização formal, sociologia quantitativa, estratificação e mobilidade estavam em crescimento, enquanto especialidades como sociologia aplicada, crime e desvio, sociologia industrial, sociologia rural e casamento e família estavam em declínio. 
Nesse sentido, o mapeamento da estrutura de especialidades de uma disciplina científica é importante porque permite investigar a natureza dos laços sociais entre as especialidades do conhecimento e o processo e a direção da difusão do conhecimento de uma área da ciência para outra, pois, como alguns estudos têm mostrado, existe um problema persistente de relacionamento entre estruturas sociais e cognitivas na ciência (Sther e Larson, 1972; Turner e Turner, 1990). Por isso, entender a recepção que especialidades dão a um conhecimento tem importância social e científica.

Por diversas razões, não é incomum que o desenvolvimento de um novo conhecimento científico não seja bem recebido pela maioria das especialidades de uma disciplina. Por exemplo, historicamente, a maioria dos sociólogos sempre teve resistências em relação à incorporação de variáveis biológicas na explicação do comportamento social, fato sustentado por dois estudos dos anos de 1990 (Ennis, 1992; Sanderson \& Ellis, 1992).

Quando investigou o padrão de conexões entre especialidades sociológicas, Ennis (1992) constatou que a especialidade denominada biossociologia aparecia isolada. Sanderson e Ellis (1992) solicitaram a uma amostra de 500 sociólogos da Associação Americana de Sociologia (ASA) que indicassem duas especialidades com as quais se identificassem. Dentre as 41 especialidades indicadas, $1,9 \%$ escolheram a sociobiologia como principal identificação e apenas 2,5\% como secundária, o que surpreendeu Sanderson e Ellis (1992). Buscando entender a importância que os sociólogos davam a variáveis biológicas, descobriram que os sociólogos norte-americanos tinham uma postura altamente antibiológica, especialmente se consideramos apenas fatores genéticos. Lacerda (2009) encontrou situação parecida quando investigou dois periódicos tradicionais de sociologia dos Estados Unidos, o American Sociological Review e o American Journal of Sociology, entre 1960 e 1998.

Os tempos mudaram e o que era considerado quase consensualmente como uma resistência metodológica agora tem sido apontado como biofobia (Sanderson, 2014; Walsh, 1995). A ciência biológica de maneira geral e a biologia evolucionista de maneira particular têm experimentado um grande desenvolvimento nas últimas décadas, a ponto de alguns sociólogos, especialmente os estadunidenses, sugerirem que a sociologia não pode, hoje, prescindir do conhecimento biológico (Ferreira, 2000; Kalkhoff et al., 2012; Schutt, Seidman, \& Keshavan, 2015; Turner \& Machalek, 2018; Walsh, 1995).

Quando uma disciplina cresce e se desenvolve, subdisciplinas ou novas especialidades emergem, às vezes, em função da interação de especialidades de uma disciplina com especialidades de outras. Por exemplo, a criação do grupo de trabalho Evolução, Biologia e Sociedade, dentro da Associação Americana de Sociologia, é sintomática desse fenômeno, pois pode ser vista como uma evidência da influência da biologia no campo sociológico, isto é, de que algumas especialidades da sociologia têm desenvolvido recombinações com o campo da biologia.

Se as disciplinas científicas forem vistas como territórios, tomando uma metáfora cartográfica, as subdisciplinas podem ser pensadas como regiões dentro desses territórios. Nesse sentido, o território da sociologia é amplo e abrange várias regiões, desde as mais centrais, caracterizadas pelo conhecimento mais generalista, às áreas fronteiriças, em que sociólogos desenvolvem pesquisa interdisciplinar. Ennis (1992), ao analisar o campo das especialidades sociológicas como uma estrutura social, encontrou padrões de agrupamento que vinculavam as especialidades, examinou a posição estrutural de especialidades particulares e considerou suas implicações para o campo sociológico como um todo.

Na estrutura social das especialidades desenhada por Ennis (1992), a especialidade sociológica aberta ao diálogo com a biologia, a biossociologia, encontrava-se em posição periférica. Contudo, a repercussão da sociobiologia a partir do final dos anos de 1970 possibilitou que algumas recombinações entre especialidades sociológicas e da biologia florescessem, a despeito das resistências da comunidade de sociólogos.

A existência de uma especialidade chamada sociologia evolucionista é hoje uma realidade (Sanderson, 2014; Turner \& Machalek, 2018). Conforme apontou Sther (1975), os membros de uma especialidade desenvolvem laços sociais com outras especialidades dentro da mesma disciplina ou recombinam-se com especialidades de outras disciplinas, formando subespecialidades. Assim, mais recentemente, sociólogos evolucionistas têm desenvolvido recombinações com especialidades da neurociência e uma nova especialidade tem se desenvolvido, a neurossociologia.

O desenvolvimento da neurociência tem feito surgir, por exemplo, a neurociência social, que tem produzido recombinações entre especialidades das 
disciplinas de psiquiatria, psicologia e neurociência. Tais recombinações tematizam a influência das estruturas sociais e a sociabilidade, temas nos quais os sociólogos tradicionalmente se concentram, o que tem levado a um engajamento de algumas especialidades da disciplina da sociologia com o campo da biologia (Schutt et al., 2015).

Quando tratam de recombinações entre especialidades da sociologia e da biologia, Schutt et al. (2015) referem-se a alguns sociólogos que estão começando a conectar a neurociência social a processos e problema sociais mais amplos, e ao desenvolvimento do que tem sido chamado de neurossociologia, ou seja, uma especialidade sociológica que investigaria cientificamente as bases neurológicas da sociabilidade humana. Em contraste com a neurociência social, um campo interdisciplinar dedicado à compreensão de como os sistemas biológicos implementam os processos sociais e o comportamento do indivíduo, a neurossociologia pretende entender como esses sistemas biológicos e comportamentos individuais ou coletivos afetam a sociedade.

Franks (2010) vê um enorme potencial para fertilização cruzada entre os campos disciplinares da sociologia e da neurociência e aponta a psicologia social como a especialidade sociológica que mais se beneficiaria com esse diálogo, mas ele não menciona trabalhos na psicologia social que estejam fazendo isso, apenas formula hipóteses sobre um cenário em que isso pudesse acontecer. Von Scheve (2003) constatou, no entanto, que a sociologia tem estado ausente das discussões sobre a "nova ciência do cérebro". Ele fez sugestões de como a neurociência pode ajudar a refinar conceitos macrossociológicos, mas vê armadilhas metodológicas e epistemológicas em uma perspectiva como a neurossociologia, embora reconheça que ela tem se desenvolvido (Von Scheve, 2003, 2011, 2018).

A neurossociologia pode, portanto, ser vista como uma especialidade sensível às repercussões da neurociência (Kalkhoff, Thye, \& Lawler, 2012), mas que outras especialidades da sociologia poderiam estar desenvolvendo recombinações com a neurociência? Por indicação da literatura sociológica, a sociologia cognitiva parece ter se tornado um campo de crescente interesse para sociólogos nos Estados Unidos, o que não constitui surpresa em um contexto de crescimento de influência da neurociência, pois cognição enquanto processo social tem sido um tema persistente na teoria sociológica (DiMaggio, 1997; Cerulo, 2010).
O tema da cognição é considerado fundamental para o entendimento de conceitos sociológicos centrais (Bergesen, 2004a, 2004b). Desde o artigo de DiMaggio (1997), que fez um balanço das consequências da revolução cognitiva para a sociologia de maneira geral e para a sociologia da cultura em particular, uma série de artigos tem discutido a relação entre sociologia e cognição (Cerulo, 2010; Bergesen, 2004a, 2004b; Heimer, 2001; Ignatow, 2007, 2012; Martin, 2010; Mather, 2010). Portanto, por indicação da literatura em sociologia, parece razoável supor que a influência da neurociência no campo sociológico pode ser identificada a partir dos campos da neurossociologia e da sociologia cognitiva.

O objetivo deste trabalho é investigar que impacto, do ponto de vista da estrutura social das especialidades sociológicas, a neurociência tem gerado na sociologia. Quais são as especialidades com as quais tem se recombinado? Que especialidades manifestam resistência a ela? As especialidades com as quais tem estabelecido vínculos são mais centrais ou mais periféricas no campo sociológico? Responder as essas perguntas pode ser útil para entender o percurso teórico e metodológico da nova especialidade, bem como o perfil de recrutados pelo campo da sociologia.

Um caminho viável para investigar a influência da neurociência nas duas subáreas sociológicas é realizar uma revisão sistemática, processo padronizado de busca de literatura, o que permitiria encontrar prováveis artigos, resenhas ou livros em que haja diálogo entre as duas especialidades e a neurociência. Ou seja, seria possível saber o que os sociólogos têm pensado a respeito da neurociência em suas produções científicas.

Para acessar o que os sociólogos têm produzido em termos de produção científica de uma maneira sistemática, um recurso que tem se mostrado valioso e factível é o processo de revisão de literatura chamado revisão sistemática. Embora não seja ainda um recurso popular na sociologia, devido a restrições que serão abordadas a seguir, a revisão sistemática tem sido adotada como um recurso de revisão da literatura em áreas que têm proximidade com a sociologia, como a psicologia, a administração e a economia.

O conceito de revisão sistemática tem sido contrastado com o conceito de revisão narrativa. Conforme Tranfield (Apud Bryman, 2016, p. 99),

Systematic review has been defined as a replicable, scientific and transparent process...that aims 
to minimize bias through exhaustive literature searchs of published and unspublished studies and by providing an audit trail of the reviewer's decisions, procedures and conclusions.

A revisão narrativa é um exame da teoria e da pesquisa relacionadas a um campo de interesse que delineia o que já é conhecido e que enquadra e justifica sua(s) pergunta(s) de pesquisa. Com a revisão narrativa, procura-se obter uma visão geral de um campo de estudo por meio de uma avaliação crítica razoavelmente abrangente e interpretativa da literatura. Um dos seus principais problemas é que ela não permite que sua construção seja auditável e, portanto, tende a ser muito influenciável pelo viés do pesquisador.

Entretanto, quando se trata do campo da sociologia, defensores da revisão sistemática reconhecem que, diferentemente das ciências médicas, em que a revisão sistemática é comum e muito considerada, o campo das ciências sociais é frequentemente caracterizado por baixo consenso sobre questões-chave de pesquisa devido à pluralidade de abordagens teóricas. Mas existem adaptações que permitem seu uso, conforme explica Bryman (2016).

\section{Método}

Bryman (2016) argumenta que as execuções dos processos de revisão sistemática podem variar ligeiramente, mas, em geral, podem ser segmentadas em cinco estágios, de uma maneira que parece contornar as restrições apontadas pelos críticos da utilização da revisão sistemática na sociologia. Os cinco estágios podem ser assim definidos:

1. Contempla a definição do esboço e objetivo da pesquisa, além da apresentação do protocolo;

2. Seleção de referências (textos) relevantes para o escopo, segundo os objetivos da pesquisa. Muitos textos que aparecem não são relevantes para os objetivos da pesquisa e, portanto, terão que ser descartados, como será mostrado nos resultados;

3. Avalia a relevância de cada estudo para a questão de pesquisa com base em suas palavras-chave;

4. Avalia a qualidade dos estudos selecionados no estágio 3. Este estágio necessita de uma especificação do critério de qualidade, tais como: se o desenho de pesquisa é adequado, se os métodos de pesquisa foram usados e se os desenho e métodos de pesquisa escolhidos foram executados de acordo com a boa prática de pesquisa;

5. Extrai os resultados de cada pesquisa e os sintetiza.
Um protocolo formal deve ser usado para registrar características, tais como: quando a pesquisa foi realizada, localização, tamanho da amostra, método de coleta de dados e principais descobertas, conforme Bryman (2016).

O primeiro estágio pode ser considerado como o do estabelecimento do protocolo. Ele define os objetivos e escopo da pesquisa e elege algumas palavras-chave. No caso em questão, o objetivo da pesquisa foi investigar se o desenvolvimento da neurociência tem gerado algum tipo de repercussão no campo disciplinar da sociologia, basicamente em duas subáreas, neurossociologia e sociologia cognitiva.

Como protocolo de busca, elegeu-se três bases de dados: Sociological Abstract, Periódicos Capes e Sage Journal Online, nas quais buscou-se apenas livros e artigos. Os livros aparecem em resenhas publicadas nos periódicos. Artigos que não estavam disponíveis na íntegra foram descartados. Sociological Abstract foi escolhido enquanto base de dados por ser a principal coleção indexadora da produção sociológica em língua inglesa. Já o Periódicos Capes foi escolhido por ser uma base de dados mais ampla que contemplaria o que Bryman (2016) chama de literatura cinza, que neste caso basicamente corresponde a livros. Finalmente, o Sage Journal Online foi selecionado por ter um portfólio de mais de 1000 periódicos e por indexar diferentes especialidades que dialogam com especialidades biológicas em geral e a neurociência em particular.

A busca foi realizada em textos em inglês, publicados nos últimos 10 anos e que incluíssem materiais como artigos e livros publicados em periódicos de sociologia ou áreas afins, vinculados a pesquisas sociológicas. O processo de busca foi feito sem o uso de filtros, seja só no título, resumo ou nas palavras-chave. As seguintes strings de busca foram sugeridas: neurosociology, sociology and neuroscience, neuroscience and human sociality, mind and social structure, mind and societye cognitive sociology and neuroscience.

As seis strings de busca foram testadas nas três bases de busca e foram excluídas as que não se revelaram apropriadas para certas bases por nada produzirem. As strings que apresentaram resultados nas bases pesquisadas estão ordenadas a seguir conforme data de busca:

a) Neurosociology (Sociological Abstract), acessada em 16/05/2018 e 17/05/2018;

b) "Cognitive sociology" AND Neuroscience (SociologicalAbstract), acessada entre 21/05/2018 e 22/05/2018; 
c) Neuroscience AND human sociality (Periódicos Capes), acessada em 18/05/2018;

d) Sociology AND neuroscience (Periódicos Capes), acessada em 22/05/2018 e 23/05/2018;

e) Mind AND "social structure" (Periódicos Capes), acessada entre 04/06/2018 e 05/06/2018;

f) Mind AND Society (Periódicos Capes), acessada em 06/06/2018;

g) Sociology AND Neuroscience (Sage Journal Online), acessada em 18/06/2018.

\section{Resultados}

O processo de busca nas três bases de dados e as fases em que a quantidade de artigos foi filtrada segundo os critérios de inclusão/exclusão desenhados no protocolo de cada etapa foram sintetizados na Tabela 1.

$\mathrm{Na}$ fase dois, efetuou-se a primeira seleção de artigos de acordo com os objetivos da pesquisa. $\mathrm{Na}$ fase três, foram descartadas as referências: a) que não fossem publicações vinculadas ao campo disciplinar da sociologia; b) que não fossem consideradas biossociais no sentido de que constituíssem diálogos entre as especialidades da sociologia e neurociência; e c) que não disponibilizassem os textos, porque muitas vezes o resumo do artigo estava disponível em inglês, mas ou o artigo completo estava em outra língua ou não estava disponível.

Finalmente, na fase quatro, avaliou-se a qualidade dos estudos. Este estágio partiu de uma especificação do critério de qualidade, tal como: se o desenho de pesquisa era adequado, se os métodos de pesquisa foram usados e se o desenho e métodos de pesquisa escolhidos foram executados de acordo com a boa prática de pesquisa. Em termos dos objetivos da pesquisa, assim traduz-se esses critérios: o texto constitui um diálogo da sociologia com as neurociências e faz considerações sobre as repercussões de descobertas na neurociência em termos sociológicos? Os métodos de pesquisa utilizados refletem isso? O texto se refere à relação da sociologia com a neurociência?

Dos 30 textos encontrados na fase quatro, a classificação final contemplou a 25, pois cinco textos eram repetidos. Na Tabela 2, listamos todos os 30 textos conforme a string a base de dados em que foram encontrados.

Tabela 1

Fases 1, 2, 3 e 4 da Revisão Sistemática segundo Bryman (2016).

\begin{tabular}{|c|c|c|c|c|c|}
\hline Palavras-Chave & Base de Dados & Fase 1 & Fase 2 & Fase 3 & Fase 4 \\
\hline Neurosociology & $\begin{array}{l}\text { Sociological } \\
\text { Abstract }\end{array}$ & 42 & 24 & 17 & 12 \\
\hline $\begin{array}{l}\text { "Cognitive Sociology" } \\
\text { AND } \\
\text { Neuroscience }\end{array}$ & $\begin{array}{l}\text { Sociological } \\
\text { Abstract }\end{array}$ & 26 & 10 & 08 & 05 \\
\hline $\begin{array}{l}\text { Sociology } \\
\text { AND } \\
\text { Neuroscience }\end{array}$ & $\begin{array}{l}\text { Periódicos } \\
\text { Capes }\end{array}$ & 88 & 25 & 10 & 10 \\
\hline $\begin{array}{l}\text { Mind } \\
\text { AND } \\
\text { "Social Structure" }\end{array}$ & $\begin{array}{l}\text { Periódicos } \\
\text { Capes }\end{array}$ & 24 & 13 & 02 & 01 \\
\hline $\begin{array}{l}\text { Mind } \\
\text { AND } \\
\text { Society }\end{array}$ & $\begin{array}{l}\text { Periódicos } \\
\text { Capes }\end{array}$ & 46 & 02 & 0 & - \\
\hline $\begin{array}{l}\text { Neuroscience } \\
\text { AND } \\
\text { Human sociality }\end{array}$ & $\begin{array}{l}\text { Periódicos } \\
\text { Capes }\end{array}$ & 10 & 0 & - & - \\
\hline $\begin{array}{l}\text { Sociology } \\
\text { AND } \\
\text { Neuroscience }\end{array}$ & $\begin{array}{l}\text { Sage Journal } \\
\text { Online }\end{array}$ & 134 & 11 & 05 & 02 \\
\hline Total & 7 & 370 & 85 & 42 & 30 \\
\hline
\end{tabular}


Tabela 2

Artigos selecionados segundo string e base de dados.

\begin{tabular}{ll}
\hline String: Neurosociology (Sociological Abstract): 12 & $\begin{array}{l}\text { String: Sociology AND Neuroscience (Periódico } \\
\text { Capes): 10 }\end{array}$ \\
\hline Firat e Hitlin (2012); & Fitzgerald, Rose e Singh (2016a); \\
Kalkhoff, Thye e Pollock (2016); & Meloni (2014a); \\
Williams (2011); & Sahni (2012); \\
Smith-Lovin e Winkielman (2010); & Shalin (2017); \\
Shalin (2017); & Fitzgerald, Rose e Singh (2016b); \\
Bone (2014); & Potter, Mashburn e Grissmer (2013); \\
Meloni (2014a); & Pickersgill (2013); \\
Simons e Klopack (2015); & Ray (2016); \\
Dunn (2013); & Shkurko (2012). \\
Tibbetts (2016); & \\
Shkurko (2012); & \\
Bone (2016). & \\
\hline String: Sociology AND Neuroscience (SAGE Journals & String: “Cognitive sociology” AND Neuroscience \\
Online): 02 & (Sociological Abstract): 05 \\
\hline Pickersgill (2013); & Sepulvado e Lizardo (2017); \\
Melamed, Kalkhoff, Han e Li (2017). & Cerulo (2010); \\
& Zerubavel e Smith (2010); \\
& Abrantes (2016); \\
\hline String: Mind and Social Structure (Periódicos Capes): 01 & Pitts-Taylor (2015). \\
\hline Bone (2016). & \\
\hline
\end{tabular}

\section{Discussão dos Resultados}

Antes de proceder à análise do conteúdo dos artigos, eles foram agrupados segundo duas questões importantes. A primeira procura saber a que especialidade da sociologia o artigo ou texto está vinculado. O resultado mostra uma heterogeneidade de especialidades, mas com concentração, em ordem decrescente, em neurossociologia, psicologia social sociológica, teoria social e sociologia cognitiva. Se, por um lado, essa ordem mostra que a neurociência tem sido invocada em várias especialidades sociológicas, por outro, mostra que a sociologia cognitiva não é uma das linhas de frente de recepção da neurociência, conforme a hipótese formulada defende. A Tabela 3 apresenta as especialidades encontradas.

Tabela 3

Especialidades da sociologia a que o artigo se vincula.

\begin{tabular}{lc} 
Especialidade Sociológica & Quantidade de Artigos \\
\hline Neurossociologia & 07 \\
Psicologia Social & 06 \\
Sociológica & 04 \\
Teoria Social & continua...
\end{tabular}

...continuação

\begin{tabular}{lc}
\hline Especialidade Sociológica & Quantidade de Artigos \\
\hline Sociologia Cognitiva & 03 \\
Sociologia Urbana & 02 \\
Sociologia da Violência & 01 \\
Sociologia da Família/ & 01 \\
Estratificação & 01 \\
Sociologia da Saúde & 25 \\
Total & \\
\hline
\end{tabular}

A moderna neurociência social baseia-se na crença de que não é mais necessário nem apropriado escolher entre o entendimento puramente biológico ou sociológico da mente humana (Schutt et al., 2015), pois o que se tem descoberto acerca do cérebro é sua capacidade de processar informações, adaptar-se e efetuar mudanças no ambiente. Essas descobertas implicam reajustes em certas versões de algumas das tradições sociológicas. Nesse sentido, quase todos os artigos posicionam-se em relação à possibilidade de desenvolver recombinações entre especialidades da sociologia e da neurociência ou áreas afins. A organização dos artigos nesse sentido consta na Tabela 4 . 


\section{Tabela 4}

Especialidades com que se propõe recombinações com a sociologia.

\begin{tabular}{|c|c|}
\hline $\begin{array}{l}\text { Recombinação entre } \\
\text { quais especialidades }\end{array}$ & $\begin{array}{l}\text { Quantidade de } \\
\text { Artigos }\end{array}$ \\
\hline $\begin{array}{l}\text { Neurossociologia/ } \\
\text { Neurociência }\end{array}$ & 13 \\
\hline $\begin{array}{l}\text { Psicologia Social } \\
\text { Sociológica/Neurociência }\end{array}$ & 03 \\
\hline $\begin{array}{l}\text { Psicologia Social } \\
\text { Sociológica/Psicologia } \\
\text { Cognitiva }\end{array}$ & 03 \\
\hline $\begin{array}{l}\text { Sociologia Cognitiva/ } \\
\text { Psicologia Cognitiva }\end{array}$ & 02 \\
\hline Sociologia/Epigenética & 02 \\
\hline $\begin{array}{l}\text { Sociologia Cognitiva/ } \\
\text { Neurociência }\end{array}$ & 01 \\
\hline Não propõe & 01 \\
\hline Total & 25 \\
\hline
\end{tabular}

Os dados mostram que as possibilidades de recombinação entre a neurossociologia e a neurociência é o tipo de diálogo interdisciplinar dominante. Os textos vinculados à neurossociologia propõem claramente a necessidade de um intercâmbio entre a sociologia, a biologia e a neurociência, o que pode demonstrar uma perspectiva biossocial.

Na mesma linha - mas ressaltando o fato de que a biologia tem se tornado mais social e os impactos disso na teoria sociológica, na medida em que constituem contraposições a abordagens mais biologizantes - estão os artigos que defendem recombinações da sociologia com a epigenética e da sociologia cognitiva com a neurociência (Fitzgerald, Rose, \& Singh, 2016a, 2016b; Meloni, 2014b; Simon \& Klopack, 2015). A maior parte dos artigos vinculados à sociologia cognitiva (dois de três) é resistente à incorporação de variáveis biológicas na explicação sociológica e, portanto, tende a incorporar descobertas da neurociência a partir de interpretações sociológicas tradicionais (Sepulvado \& Lizardo, 2017; Zerubavel \& Smith, 2010).

Da mesma forma, metade dos textos categorizados como psicologia social sociológica também mostra certa resistência para recombinações biossociais. Situados na tradição sociológica denominada interacionismo simbólico, eles rejeitam a concepção positivista de ciência e qualquer incorporação de variáveis biológicas nas explicações sociológicas (Sahni, 2012; Shalin, 2017; Smith-Lovin \& Winkielman, 2010;). Já a outra metade da psicologia social sociológica defende a necessidade de se rever conceitos sociológicos, pois eles precisariam ser atualizados frente às descobertas da neurociência (Dunn, 2013; Melamed, Kalkhoff, Han, \& Li, 2017; Fitzgerald et al., 2016a).

Além da importância de levar em consideração os avanços da neurociência, outra ideia que aparece nos textos simpáticos a um diálogo com a neurociência é a de que esse diálogo envolve psicologia social e nela desemboca (Melamed et al., 2017; Smith-Lovin \& Winkielman, 2010; Williams, 2011). Se em parte dos textos existe a sugestão de que os conceitos tradicionais da psicologia social e sociologia dão conta dos desafios apresentados pela neurociência, em outra parte são recorrentes as ideias de que alguns conceitos sociológicos precisam ser reformulados, atualizados, e de que um diálogo com a psicologia evolucionista, neurociência e outras abordagens biossociais é imprescindível (Shkurko, 2012; Meloni, 2014a, 2014b; Simons \& Klopack, 2015; Kalkhoff, Thye, \& Pollock, 2016; Ray, 2016; Fitzgerald et al., 2016a).

Entre os temas e conceitos mais recorrentes nos artigos, figuram, em ordem de importância: a relação entre estrutura social e emoções; o uso de técnicas da neurociência para relacionar as atividades neurológicas com as interações sociais; a repercussão do conceito de neurônio espelho para conceitos sociológicos básicos; a hipótese do cérebro social; repercussões das suposições da epigenética e a ideia de neuroplasticidade para entender a influência social sobre o cérebro; a hipótese da mente estendida; e a influência de descobertas da neurociência sobre as noções de self e identidade.

Esses temas e conceitos podem ser mais bem entendidos quando descritos segundo as estratégias de recombinação sociologia-neurociência. Três estratégias de recombinações entre a sociologia e a neurociência foram identificadas no conjunto de textos: a) processa as informações e descobertas da neurociência a partir do que a interpretação sociológica entende ser importante, mas se opõe ao uso de conceitos ou abordagens que considere importante a incorporação de variáveis biológicas, como as abordagens biossociais, por exemplo; b) processa as informações e descobertas da neurociência a partir de descobertas ou suposições de teorias que defendem que a influência social sobre o cérebro e sobre a biologia de maneira geral é mais acentuada do que se pensava, 
o que revigora e restabelece a importância da sociologia para a neurociência; c) processa as informações e descobertas da neurociência a partir da ideia de que é necessário repensar alguns conceitos sociológicos, bem como a relação entre a sociologia e a biologia, e reformular conceitos sociológicos em uma perspectiva biossocial.

A primeira estratégia tende a enfatizar alguns conceitos e temas a partir de perspectivas sociológicas mais resistentes a recombinações entre a sociologia e a neurociência, e entre a sociologia e a biologia. A tradição do interacionismo simbólico presente nas perspectivas da psicologia social sociológica, da sociologia cognitiva e da sociologia que dialoga com o pensamento feminista tende a defender esse tipo de estratégia (Sahni, 2012; Shalin, 2017; Pitts-Taylor, 2015; Smith-Lovin \& Winkielman, 2010). Nela sobressaem temas como a importância das emoções para entender o self, a agência humana; e a hipótese da mente estendida, que afirma não existir um limite identificável para os processos cognitivos, que frequentemente se estendem para o ambiente, ou seja, defende a ideia de que o ambiente possui papel ativo nos processos cognitivos. A discussão da hipótese da mente estendida, conforme Clark e Chalmers (1998), tem sido conectada a suposições da epigenética de que mesmo a expressão gênica, em vez de ser um processo constitutivo direto, também é altamente sensível ao meio ambiente. Assim, existiria transmissão de experiências ocorridas com os pais para os filhos, a despeito de isso não ser feito por meio de um DNA que ignora o ambiente. Outrossim, uma sociologia interacionista fundamentada em George Mead e Vygotsky tende a explorar a ideia de que o genoma é social (Shalin, 2017). Alguns sociólogos tendem a enxergar na epigenética uma indicação de que o ambiente social teria mais importância do que certas explicações biológicas estariam dispostas a reconhecer (Fitzgerald et al., 2016a, 2016b; Simons \& Klopack, 2015).

A segunda estratégia considera as suposições e descobertas da epigenética como indicadoras de mudanças que têm acontecido na biologia e que constituem aberturas para disciplinas como a sociologia. Os métodos e tecnologia da neurociência para estudar atividade no cérebro, como a imagem por ressonância magnética funcional (fMRI, Functional Magnetic Ressonance Imaging), técnica específica capaz de detectar variações no fluxo sanguíneo em resposta à atividade neural, e o eletroencefalograma (EEG), método de monitoramento eletrofisiológico que pode detectar diversos problemas neurológicos como lesões e tumores, são apontados como recursos importantes que permitiriam investigar, por exemplo, como a "coordenação cerebral" representa relações emergentes de coesão social em trocas diádicas (Kalkhoff et al., 2016; Melamed et al., 2017; Ray, 2016; Simons \& Klopack, 2015). Os estudos neurobiológicos das emoções podem ser atrelados a estudos sociológicos que relacionam emoções ao comportamento social. As perspectivas da psicologia social sociológica e algumas propostas de recombinações entre a sociologia e a neurociência tendem a enfatizar a segunda estratégia, conforme apresenta a Tabela 4 .

Finalmente, a terceira estratégia propõe recombinações entre a sociologia e a neurociência a partir de mudanças que devem ocorrer na maneira como os sociólogos olham para variáveis biológicas. É nesse sentido que são abordados a hipótese do cérebro social, o uso de técnicas da neurociência, o conceito de neurônio espelho e a temática das emoções. A especialidade da neurossociologia tem destaque nesse tipo de estratégia, mas é possível encontrá-la também em certas abordagens da sociologia cognitiva e da psicologia social sociológica. Aqui, a epigenética é abordada por meio de mecanismos como a metilação, em que fatores ambientais transitórios podem deixar um traço biológico e se tornar parte da memória do próprio material genético. Os estudos epigenéticos mencionados se referem à literatura da teoria evolucionista, que discute mutações epigenéticas como casos de plasticidade de desenvolvimento e estudos epigenéticos em neurociência e epidemiologia. Os estudos epigenéticos são apresentados como indicadores de que alguns conceitos sociológicos precisam ser reformulados numa perspectiva biossocial para serem consistentes com a descobertas da epigenética (Kalkhoff et al., 2016; Meloni, 2014a, 2014b; Pickersgill, 2013; Shkurko, 2012; Simons \& Klopack, 2015; Tibbetts, 2016; Williams, 2011).

\section{Conclusão}

Em resposta à pergunta que inspirou esta pesquisa, sobre a hipótese de o desenvolvimento da neurociência ter gerado repercussões na sociologia, pode-se dizer que sim. Existem algumas repercussões no campo sociológico, que basicamente têm se materializado em antigas e novas especialidades sociológicas. Mas, diferentemente da hipótese que foi formulada, 
a sociologia cognitiva não é protagonista desse exercício, apesar de seu desenvolvimento recente nos EUA e de figurar entre as especialidades que mais têm fomentado diálogo entre a sociologia e a neurociência.

Além da neurossociologia, especialidade recente, a subárea da psicologia social sociológica parece ser um campo aberto à recepção das descobertas da neurociência na sociologia. As interfaces entre a neurossociologia e a neurociência e entre a sociologia cognitiva e a neurociência tendem, pelo que os textos encontrados indicam, a apresentar temáticas de interesse comuns em áreas como atenção, percepção, tomada de decisão, memória, moralidade e emoção. Parte dos sociólogos cognitivos faz esse diálogo com a psicologia cognitiva, na maioria das vezes por meio de uma interpretação sociológica tradicional. Os neurossociólogos, ao contrário, partem do pressuposto de que o conhecimento sociológico tradicional precisa ser atualizado, reformulado, e que pontes entre as ciências sociais e a neurociência, entre a sociologia e áreas da biologia, como o pensamento evolucionista, precisam ser construídas.

A repercussão da neurociência no campo sociológico, portanto, pode ser encontrada em duas especialidades, uma mais recente, a neurossociologia, e a outra mais tradicional, a psicologia social. Uma especialidade, a psicologia social, é mais central na estrutura social do campo sociológico, e a neurossociologia, mais periférica, tem se desenhado a partir de recombinações com especialidades que discutem proximidades entre a sociologia e as ciências biológicas.

A localização das especialidades que têm repercutido os avanços da neurociência na sociologia permite também conhecer os temas abordados e seus principais conceitos. Referências à neurociência social não foram significativas nos textos encontrados, talvez porque exista um esforço para demarcar uma dimensão sociológica da temática. Existe a insistência em muitos textos de que alguns conceitos sociológicos são importantes (como socialização, estratificação social e status) e outros tantos precisam ser reformulados para, à luz da neurociência, ter-se um melhor entendimento de temas como violência e urbanização.
A relação entre a sociologia e a neurociência é recente. A neurossociologia é um campo novo e, dentro dos Grupos de Trabalho da Associação Americana de Sociologia, localiza-se no GT Evolução, Biologia e Sociedade, grupo que congrega os sociólogos interessados em discutir as repercussões da teoria evolucionista na sociologia. Por essa localização, uma hipótese a ser explorada é de que a neurossociologia tende a criar uma maior proximidade com a neurociência, na medida em que partilham interesses teóricos e metodológicos, ao contrário de certas versões da sociologia cognitiva que rejeitam a incorporação de variáveis biológicas em suas explicações, como faz a sociologia cognitiva popular de Zerubavel (1996).

A psicologia social sociológica tem também um GT na Associação Americana de Sociologia. É uma especialidade bem estabelecida que divide com a psicologia social psicológica a investigação de temas como socialização, atitudes e relações interpessoais, temas considerados no Brasil como de mais interesse de psicólogos do que de sociólogos. Uma hipótese quanto ao desenvolvimento da relação entre a sociologia e a neurociência nessa especialidade é a de que o diálogo com a neurociência passará fundamentalmente pela psicologia, na medida em que, nos cinco textos encontrados, o diálogo com a neurociência passa pela psicologia cognitiva.

Este trabalho teve caráter exploratório. Novas investigações são necessárias para se entender melhor como o desenvolvimento da neurociência em geral e da neurociência social em particular tem repercutido no campo sociológico. As especialidades sociológicas estabelecerão proximidade com a neurociência social ou tentarão se diferenciar, como parece estar acontecendo? A sociologia nos Estados Unidos se desenvolveu a partir de um laço forte com a psicologia, o que ainda hoje se faz presente em uma das tradições sociológicas, o interacionismo simbólico, justamente a tradição que se opõe ao desenvolvimento de recombinações entre especialidades da sociologia e da neurociência em um caminho biossocial. No futuro, verificar-se-á se esse laço será ampliado ou redefinido a partir da interação da neurociência com outras especialidades sociológicas.

\section{Referências}

Abrantes, P. (2016). Socialização: Onde a sociologia e as neurociências se encontram. Sociologia, Problemas $e$ Práticas, (82), 11-25.

Bergesen, A. J. (2004a). Chomsky versus Mead. Sociological Theory, 22(3), 357-370.

Bergesen, A. J. (2004b). Durkheim's theory of mental category: A review of the evidence. Annual Review of Sociology, 30, 395-408. https://doi.org/10.1146/annurev.soc.30.012703.110549 
Bone, J. (2014). Neoliberal Nomads: Housing insecurity and the revival of private renting in the UK. Sociological Research Online, 19(4), 1-14. https://doi.org/10.5153/sro.3491

Bone, J. (2016). The Nature of Structure: a biosocial approach. The Sociological Review Monographs, 64(1), 238-255. https://doi.org/10.1002/2059-7932.12023

Bryman, A. (2016). Social Research Methods. Oxford University Press.

Cerulo, K. A. (2010). Mining the intersections of cognitive sociology and neuroscience. Poetics, 38(2), 115-132. https:// doi.org/10.1016/j.poetic.2009.11.005

Clark, A., \& Chalmers, D. (1998). The Extended Mind. Analysis, 58(1), 7-19.

DiMaggio, P. (1997). Culture and cognition. Annual Review of Sociology, 23, 263-287. https://doi.org/10.1146/ annurev.soc.23.1.263

Dunn, J. L. (2013). The Problem of Emotions in Societies. Teaching Sociology, 41(2), 128-129.

Ennis, J. G. (1992). The Social Organization of Sociological Knowledge: Modeling the Intersection of Specialties. American Sociological Review, 57,(2), 259-265.

Ferreira, A. L. R. (2000). Sob o Espectro de Darwin: a sociologia e as abordagens biossociais. [Tese de Doutorado, Departamento de Sociologia, Universidade de Brasília].

Firat, R., \& Hitlin, S. (2012). Neurosociology and the Dificult Art of Building Interdisciplinary Bridges. Contemporary Sociology, 41(6), 780-783. https://doi.org/10.1177/0094306112462559a

Fitzgerald, D., Rose, N., \& Singh, I. (2016a). Living well in the Neuropolis, The Sociological Review Monographs, 64(1), 221-237. https://doi.org/10.1002/2059-7932.12022

Fitzgerald, D., Rose, N., \& Singh, I. (2016b). Revitalizing sociology: urban life and mental illness between history and the present. Br. J. Sociol, 67(1), 138-60. https://doi.org/10.1111/1468-4446.12188

Franks, D. D. (2010). Neurosociology: The nexus between neuroscience and social psychology. Springer.

Heimer, C. A. (2001). Cases and Biographies: An Essay on Routinization and the Nature of Comparison. Annual Review of Sociology, 27, 47-76. https://doi.org/10.1146/annurev.soc.27.1.47

Ignatow, G. (2007). Theories of embodied knowledge: new directions for cultural and cognitive sociology? Journal for the Theory of Social Behavior, 37(2), 115-135. https://doi.org/10.1111/j.1468-5914.2007.00328.x

Ignatow, G. (2012). Mauss's lectures to psychologists: A case for holistic sociology. Journal of Classical Sociology, 12(1), 3-12. https://doi.org/10.1177/1468795X11433703

Kalkhoff, W., Thye, S. R., \& Lawler, E. J. (Eds.). (2012). Advances in Group Process: Vol. 29. Biosociology and Neurosociology. Emerald Group Publishing Limited.

Kalkhoff, W., Thye, S. R, \& Pollock, J. (2016). Developments in Neurosociology. Sociology Compass, 10(3), 242-258. https:// doi.org/10.1111/soc4.12355

Lacerda, A. L. R. (2009). Abordagens biossociais na sociologia: Biossociologia ou sociologia evolucionista? Revista Brasileira de Ciências Sociais, 24(70). https:// doi.org/10.1590/S0102-69092009000200010

Martin, J. L. (2010). Life's a beach but you're an ant, and other unwelcome news for the sociology of culture. Poetics, 38(2), 228-243. https://doi.org/10.1016/j.poetic.2009.11.004

Mather, M. (2010). Aging and cognition. Cognitive Science, 1(3), 346-362. https://doi.org/10.1002/wcs.64

Melamed, D., Kalkhoff, W., Han, S., \& Li, X. (2017). The Neural Bases of Status-Based Influence. Socius: Sociological Research for a Dynamic World, 3, 1-10. https://doi.org/10.1177/2378023117709695

Meloni, M. (2014a). Biology without Biologism: social theory in a postgenomic age. Sociology, 48(4), 731-746. https://doi.org/10.1177/0038038513501944

Meloni, M. (2014b). How Biology became social, and what it means for social theory. The Sociological Review, 62(3), 593-614. https://doi.org/10.1111/1467-954X.12151

Pickersgill, M. (2013). The social life of the brain: Neuroscience in society. Current Sociology, 61(3), 322-340. https://doi.org/10.1177/0011392113476464 
Pitts-Taylor, V. (2015). A Feminist Carnal Sociology? Embodiment in Sociology, Feminism, and Naturalized Philosophy. Qualitative Sociology, 38(1), 19-25. https://doi.org/10.1007/s11133-014-9298-4

Potter, D., Mashburn, A., \& Grissmer, D. (2013). The family, neuroscience, and academic skills: An interdisciplinary account of social class gaps in children's test scores. Social Science Research, 42(2), 446-464. https://doi.org/ 10.1016/j.ssresearch.2012.09.009

Ray, L. (2016). Explaining violence - towards a critical friendship with neuroscience? Journal for the Theory of Social Behaviour, 46(3), 335-356. https://doi.org/10.1111/jtsb.12102

Sahni, I. (2012). More than a Façade: Somatic and Structural Determinants in Erving Goffman's Theory of the Perduring Self. Symbolic Interactions, 35(2), 162-185. https://doi.org/10.1002/symb.9

Sanderson, S. K., \& Ellis, E. (1992). Theoretical and political perspectives of American sociologists in the 1990S. American Sociologist, 23(2), 26-42.

Sanderson, S. K. (2014). Human Nature and the Evolution of Society. Westview Press.

Schutt, R. K., Seidman, L. J., \& Keshavan, M. S. (Eds.). (2015). Social Neuroscience: Brain, Mind, and Society. Harvard University Press.

Sepulvado, B., \& Lizardo, O. (2017). Cognitive Sociology in France. American Sociologist, 48(3/4), 366-381.

Shalin, D. (2017). Extended Mind and Embodied Social Psychology: historical perspectives. Society, 54(2), 171-186. https://doi.org/10.1007/s12115-017-0118-9

Shkurko, A. (2012). Role behavior: A neurosociological perspective. Social Science Information, 51(3), 338-363. https://doi.org/10.1177/0539018412441751

Simons, R. L., \& Klopack, E. T. (2015). Invited Address: “The Times They Are A-Changin”" gene expression, neuroplasticity, and developmental research. J Youth Adolescence, 44(3), 573-580. https://doi.org/10.1007/ s10964-014-0245-1

Smith-Lovin, L., \& Winkielman, P. (2010). The Social Psychologies of Emotion: a bridge that is not too far. Social Psychology Quarterly, 73(4), 327-332.

Stehr, N. (1975). Fators in the development of multi-paradigm disciplines: the case of sociology. Journal of the History of the Behavioral Sciences, 11(2), 172-188. https://doi.org/10.1002/1520-6696(197504)11:2<172:: AID-JHBS2300110208>3.0.CO;2-F

Sther, N., \& Larson, L. (1972). The rise and decline of areas of specialization. American Sociologist, 7(7), 4-6.

Tibbetts, P. (2016). Sociology and Neuroscience: An emerging dialogue. The American Sociologist, 47(1), 36-46.

Turner, J. H., \& Machalek, R. S. (2018). The New Evolutionary Sociology. Routledge.

Turner, S., \& Turner, J. H. (1990). The impossible science: An institutional analyze of American sociology. Sage.

Von Scheve, C. (2003). Sociology of Neuroscience or Neurosociology? Advances in Medical Sociology, 13, 255-278.

Von Scheve, C. (2011). Sociology of Neuroscience or Neurosociology? In M. Pickergill \& J. Van Keulen (Eds.), Advances in Medical Sociology: Vol. 13. Sociological Reflections on the Neurosciences (pp. 255-278). Emerald Group Publishing Limited.

Von Scheve, C. (2018). Affective neuroscience as sociological inquiry? In M. Meloni, J. Cromby, D. Fitzgerald \& S. Lloyd (Eds.), The Palgrave Handbook of Biology and Society (pp. 391-415). Palgrave Macmillan. https://www.researchgate.net/publication/323540665_Affective_neuroscience_as_sociological_inquiry

Walsh, A. (1995). Biosociology: An Emerging Paradigm. Praeger Publishers.

Williams, S. J. (2011). Neurosociology: the nexus between neuroscience and social psychology. British Journal of Sociology, 62(4), 744-745. https://doi.org/10.1111/j.1468-4446.2011.01389_4.x

Zerubavel, E. (1996). Social memories: Steps to a sociology of the past. Qualitative Sociology, 19(3), 283-299. https://doi.org/10.1007/BF02393273

Zerubavel, E., \& Smith, E. R. (2010). Transcending Cognitive Individualism. Social Psychology Quarterly, 73(4), 321-325. https://doi.org/10.1177/0190272510388998 


\section{André Ribeiro Lacerda}

Professor Titular do Departamento de Sociologia e Ciência Política da Universidade Federal do Mato Grosso, Cuiabá - MT. Brasil. Áreas de atuação: Sociologia Evolucionista, Impactos da Modernização sobre as Estruturas Socias de Famílias e Ocupações.

E-mail: ribeirolacerda66@gmail.com

(1) https://orcid.org/0000-0002-6145-4810

Endereço para envio de correspondência:

Departamento de Sociologia e Ciência Política da Universidade Federal do Mato Grosso. Avenida Fernando Correa da Costa, 2.367. Bairro Boa Esperança. CEP: 78060-900. Cuiabá - MT. Brasil.

Recebido 14/03/2019

Aceito 10/08/2020

Received 03/14/2019

Approved 08/10/2020

Recibido 14/03/2019

Aceptado 10/08/2020

Como citar: Lacerda, A. L. R. (2021). Sob o Espectro da Neurociência: a Neurossociologia, a Psicologia Social e as Abordagens Biossociais. Psicologia: Ciência e Profissão, 41, 1-13. https://doi.org/10.1590/1982-3703003221265

How to cite: Lacerda, A. L. R. (2021). Under the Neuroscience Spectrum: Neurosociology, Social Psychology and Biosocial Approaches. Psicologia: Ciência e Profissão, 41, 1-13. https://doi.org/10.1590/1982-3703003221265

Cómo citar: Lacerda, A. L. R. (2021). Bajo el Espectro de la Neurociencia: Neurosociología, Psicología Social y Enfoques Biosociales. Psicologia: Ciência e Profissão, 41, 1-13. https://doi.org/10.1590/1982-3703003221265 